\title{
Repercusión de la diabetes tipo 2 en la clasificación TNM de pacientes con cáncer renal
}

Uscanga-Yépez J, ${ }^{1}$ González-Oyervides R, ${ }^{1}$ Olvera-Posada $D,{ }^{2}$ Góngora-Cortéz $\mathrm{JJ},{ }^{3}$ Barrera Juárez $\mathrm{E}^{2}$

\section{Resumen}

OBJETIVO: Analizar la repercusión de la diabetes tipo 2 en la clasificación TNM (estadificación) de pacientes con cáncer renal y nefrectomía.

MATERIALES Y MÉTODOS: Estudio transversal, retrospectivo y descriptivo, efectuado mediante la revisión de los expedientes de pacientes con carcinoma de células renales esporádico y unilateral, nefrectomía, atendidos entre 2008 y 2014 en el Departamento de Urología del Hospital Metropolitano Dr. Bernardo Sepúlveda. Variables demográficas y clínicas: edad, sexo, tamaño tumoral patológico y tipo de cirugía (radical o parcial); comorbilidades (hipertensión arterial sistémica, diabetes mellitus tipo 2 y dislipidemia); estadificación y tamaño del tumor (clasificación TNM 2009), grado de diferenciación celular y tipo histológico. Las características clínicas y patológicas se analizaron de forma independiente con la prueba $\chi^{2}$. Los índices de supervivencia global y libre de recurrencia se analizaron con la prueba de Kaplan-Meier.

RESULTADOS: Se estudiaron 43 pacientes, $18.6 \%$ de ellos con diabetes tipo 2. Predominaron los tumores T1b en $32.6 \%$ de los casos. No se encontraron diferencias estadísticamente significativas al comparar el estadio tumoral patológico $(p=0.5)$, enfermedad ganglionar $(p=0.7)$ y metástasis $(p=0.5)$ en pacientes con y sin diabetes. La mediana de seguimiento fue de 45 meses, diferencias al comparar la supervivencia global y libre de recurrencia en pacientes con y sin diabetes tipo 2 ( $p$ $=0.76$ y 0.78 , respectivamente).

CONCLUSIÓN: La diabetes tipo 2 no repercute de forma significativa en el estadio patológico de pacientes con cáncer renal, ni en la supervivencia global ni libre de recurrencia.

PALABRAS CLAVE: Cáncer renal; clasificación TNM; nefrectomía; diabetes mellitus tipo 2 .

\footnotetext{
${ }^{1}$ Departamento de Urología, Hospital Metropolitano Dr. Bernardo Sepúlveda.

${ }^{2}$ Urólogo, Centro Médico Zambrano Hellion, Instituto de Cirugía.

${ }^{3}$ Departamento de Evaluación y Estadística.

Escuela Nacional de Medicina, Instituto de Estudios Superiores de Monterrey, Monterrey, Nuevo León, México.
}

Recibido: mayo 2017

Aceptado: noviembre 2017

Correspondencia

Dr. Jaime Uscanga Yépez

j.uscanga.y@gmail.com

Este artículo debe citarse como

Uscanga-Yépez J, González-Oyervides R, OlveraPosada D, Góngora-Cortéz JJ, Barrera-Juárez E. Repercusión de la diabetes tipo 2 en la clasificación TNM de pacientes con cáncer renal. Rev Mex Urol. 2018 ene-feb;78(1):1-8.

DOI: https://doi.org/10.24245/revmexurol.v78i1.1458 
Rev Mex Urol. 2018 Jan-Feb;78(1):1-8.

\title{
Impact of type $\mathbf{2}$ diabetes mellitus on the TNM classification of kidney cancer
}

\author{
Uscanga-Yépez J, ${ }^{1}$ González-Oyervides R, ${ }^{1}$ Olvera-Posada D, ${ }^{2}$ Góngora-Cortéz \\ $\mathrm{JJ},{ }^{3}$ Barrera Juárez $\mathrm{E}^{2}$
}

\begin{abstract}
OBJECTIVE: To evaluate the influence of type 2 diabetes mellitus on the TNM staging classification in patients with renal cell carcinoma treated through nephrectomy.

MATERIALS AND METHODS: A retrospective, cross-sectional, descriptive study was conducted. The case records were reviewed of patients with sporadic and unilateral renal cell carcinoma that underwent nephrectomy at the Urology Department of the Hospital Metropolitano Dr. Bernardo Sepúlveda. Demographic and clinical variables: age, sex, pathologic tumor size, and type of surgery (radical or partial). The comorbidities evaluated were high blood pressure, type 2 diabetes mellitus, and dyslipidemia. Staging and tumor size (2009 TNM classification), Fuhrman grade, and histologic type were analyzed. Each TNM classification result was analyzed independently through the $\chi^{2}$ test. Overall survival and recurrence-free survival were evaluated through the Kaplan-Meier test.
\end{abstract}

RESULTS: A total of $18.6 \%$ of the 43 study patients presented with type 2 diabetes mellitus. T1b tumors predominated in $32.6 \%$ of the cases. No statistically significant differences were found in the comparison of pathologic tumor stage $(p=0.5)$, lymph node disease $(p=$ $0.7)$, or metastasis $(p=0.5)$ between patients with and without diabetes. Median follow-up was 45 months and no differences were found in the comparison of overall survival and recurrence-free survival in the patients with type 2 diabetes mellitus $(p=0.76)$ and those without the pathology $(p=0.78)$.

CONCLUSIONS: Type 2 diabetes mellitus had no significant impact on the pathologic stage of renal cell carcinoma or on overall survival and recurrence-free survival in those patients.

KEYWORDS: Renal cell carcinoma; TNM classification; Nephrectomy; Type 2 diabetes mellitus.

\section{ANTECEDENTES}

El carcinoma de células renales representa 2-3\% de todas las neoplasias malignas del paciente adulto. En Estados Unidos y Europa se estima una incidencia de 3-7 y $2-3 \%$, respectivamente. ${ }^{1}$ De igual forma, en Estados Unidos se reportan
65,000 nuevos casos cada año y 35\% de mortalidad por cáncer a 5 años. ${ }^{2}$ En México, de 106,238 neoplasias registradas en 2006, se diagnosticaron 1,973 casos de cáncer renal, lo que representó una incidencia de $1.8 \% .^{3}$ Entre los factores de riesgo implicados en el carcinoma de células renales se encuentran: tabaquismo, hipertensión 
arterial sistémica, obesidad y, recientemente, diabetes mellitus tipo $2 .^{4-8} \mathrm{El}$ incremento de la prevalencia de hipertensión arterial, obesidad y diabetes tipo 2 en México y Estados Unidos se asocia con elevada incidencia de carcinoma de células renales. ${ }^{4}$ Actualmente, el sistema de clasificación TNM (tumores, nódulos y metástasis) es uno de los factores pronóstico más importantes en pacientes con cáncer renal. En la repercusión del pronóstico de la neoplasia han cobrado relevancia diversos factores metabólicos; algunos estudios ofrecen evidencia adicional en la toma de decisiones terapéuticas para incrementar la supervivencia. ${ }^{9-12}$

La diabetes tipo 2 es una enfermedad estudiada como factor de riesgo de carcinoma de células renales. ${ }^{5,13}$ La hiperinsulinemia representa un factor adicional distintivo de la diabetes tipo 2, concomitante con la sobreexpresión del IGF-1 (factor de crecimiento similar a la insulina tipo 1), citocinas inflamatorias y diversas especies de radicales libres que alteran la proliferación celular y el crecimiento tumoral. ${ }^{8}$ Psutka y sus colaboradores ${ }^{14}$ demostraron que la diabetes tipo 2 es un factor de riesgo independiente asociado con disminución de la supervivencia al cáncer, específica y global, en pacientes intervenidos quirúrgicamente de carcinoma de células renales. Lee y su grupo ${ }^{15}$ también señalan que la diabetes tipo 2 representa un factor de mal pronóstico de supervivencia global en estos pacientes. Sin embargo, Hofner y su equipo de trabajo ${ }^{16}$ reportaron que la diabetes tipo 2 no está implicada en el pronóstico del cáncer renal al momento de la cirugía, pero representa un factor de riesgo independiente de muerte.

Hasta la fecha no se ha estudiado de forma clara si la diabetes tipo 2 participa en la aparición y crecimiento del tumor y, por consiguiente, afecta el pronóstico de los pacientes con carcinoma de células renales. Por lo anterior, el objetivo de este estudio es analizar la repercusión de la diabetes tipo 2 en la clasificación TNM (estadificación) de pacientes con cáncer renal y nefrectomía.

\section{MATERIALES Y MÉTODOS}

Estudio transversal, retrospectivo y descriptivo, efectuado mediante la revisión de los expedientes de pacientes con carcinoma de células renales, esporádico y unilateral, a quienes se practicó nefrectomía entre 2008 y 2014 en el Departamento de Urología del Hospital Metropolitano Dr. Bernardo Sepúlveda (SSA) de Monterrey, Nuevo León.

Los pacientes se clasificaron con o sin diabetes tipo 2, según su estado preoperatorio. Las variables demográficas y clínicas estudiadas fueron: edad, sexo, tamaño tumoral patológico y tipo de cirugía (radical o parcial); factores de riesgo: hipertensión arterial sistémica, diabetes mellitus tipo 2 (DM2), dislipidemia y tabaquismo. Otras variables analizadas fueron los síntomas preoperatorios y la tasa de filtración glomerular, calculada mediante la fórmula MDRD (Modification of Diet in Renal Disease). ${ }^{17}$ Se consideraron hallazgos patológicos como: estadificación, incluido el tamaño tumoral (clasificación TNM de 2009 propuesta por la American Joint Committee On Cancer [AJCC]), grado de diferenciación celular (clasificación de Fuhrman) y tipo histológico, revisados por dos patólogos del departamento de Patología de la institución (Cuadro 1).

Después de la nefrectomía radical o parcial se programó el seguimiento de los pacientes, en la consulta de Urología, cada 3 o 6 meses por los primeros 2 años y cada 6 meses con evaluación radiológica, mediante tomografía computada de abdomen y pelvis contrastada, y radiografía de tórax. La detección de recurrencia local, progresión, metástasis o muerte (confirmada con el certificado de defunción) fue determinada durante el seguimiento. La supervivencia global y 
libre de recurrencia se calculó según la fecha de la cirugía y hasta la última cita del seguimiento.

Los datos se analizaron con estadística descriptiva, mediante medias y rangos intercuartiles. Los pacientes con carcinoma de células renales fueron pareados 1:2 con y sin diabetes tipo 2 , según la clasificación TNM previamente descrita, analizando cada $T, N$ y $M$ de forma independiente con la prueba de $\chi^{2}$. Los índices de supervivencia global y supervivencia libre de recurrencia se analizaron con la prueba de Kaplan-Meier, mediante la comparación de pacientes con y sin diabetes tipo 2 prequirúrgica. Para el análisis estadístico se utilizó el programa $\mathrm{SPSS}^{\circledast}$. Se consideró estadísticamente significativo el valor de $\mathrm{p}<0.05$.

\section{RESULTADOS}

Se registraron 43 pacientes con carcinoma de células renales. La media de edad fue de 57 años; hubo mayor predominio de hombres (53.5\%) que de mujeres (46.5\%). El tipo de cirugía fue seleccionada por el urólogo tratante, según el tamaño tumoral por tomografía computada y la localización del tumor; $90.7 \%$ fueron casos de nefrectomía radical. El promedio del tamaño tumoral fue de $7.2 \mathrm{~cm}$, predominando los mayores de $4 \mathrm{~cm}$ (el más grande de todos midió $16 \mathrm{~cm}$ ). Entre las características de los pacientes, se registraron $32.6 \%$ con hipertensión y $18.6 \%$ con diabetes tipo 2 . La manifestación de los tumores renales fue asintomática (haIlazgos incidentales) en $32.6 \%$ de los casos y dolor en el flanco y hematuria en otro $32.6 \%$. Por lo que se refiere a la clasificación TNM se reportaron tumores de $4-7 \mathrm{~cm}$ en $32.6 \%$ de los casos, seguido de tumores de mayor tamaño. El grado de diferenciación celular tipo II, según la clasificación de Fuhrman, predominó en 51.2\% de los pacientes. El tipo histológico identificado con mayor frecuencia fue el carcinoma de células claras (83\%) en todas las piezas quirúrgicas (Cuadro 1).
Cuadro 1. Características de los pacientes

\section{Variables}

Pacientes

Edad (años)

$57(40-76)$

Sexo (\%)

Hombre

53.5

Mujer

46.5

Tamaño tumoral* $(\mathrm{cm})$

$7.2(2.3-16)$

Tipo de nefrectomía (\%)

Radical

90.7

Parcial

9.3

Hipertensión arterial sistémica

32.6

Diabetes

18.6

Dislipidemia

7

Tabaquismo (\%)

Nunca

62.8

Frecuente

37.2

Síntomas

Incidental

32.6

Hematuria

14

Dolor en flanco

14

Otros

18.6

Estadio (\%)

pT1a 14

pT1b 32.6

pT2a

27.9

pT2b

16.3

pT3a

4.7

pT4 4.7

Grado Fuhrman (\%)

1 7

2 51.2

3 25.6

4

16.3

Histología (\%)

Células claras

83.7

Papilar

7

Cromófobo

9.3

Median $\mathrm{mL} / \mathrm{min} / 1.73 \mathrm{~m}^{2}$ TFG ${ }^{* *}$ Prequirúr-

94.5

gico

Median $\mathrm{mL} / \mathrm{min} / 1.73 \mathrm{~m}^{2}$ TFG** Posqui-

rúrgico

82.4

*Tamaño tumoral patológico,

**Cálculo según la MDRD. 
No se encontraron diferencias estadísticamente significativas al comparar el estadio tumoral patológico en pacientes con y sin diabetes tipo $2(p=0.5)$, coexistencia o no de ganglios $(p=$ 0.7 ), ni metástasis $(p=0.5)$ (Cuadro 2). La media de seguimiento fue de 45 meses. Al comparar la supervivencia global en pacientes con y sin diabetes tipo 2 se encontró 35.5 versus 44.2 meses, respectivamente, sin diferencia estadísticamente significativa $(p=0.76)$. Por su parte, la media de supervivencia libre de recurrencia en pacientes con y sin diabetes fue de 25.5 versus 28.6 meses, respectivamente, sin demostrar significación estadística en ambos grupos $(p=0.78)$ (Cuadro 3).

\section{DISCUSIÓN}

Existen pocos estudios que demuestran la repercusión de la diabetes tipo 2 en el pronóstico de pacientes con carcinoma de células renales; por tanto, la relación entre ambas variables ha llamado la atención de los investigadores en los últimos años. El aumento global de la incidencia del carcinoma de células renales y la diabetes tipo 2 ha estimulado la investigación de los desórdenes metabólicos implicados en la patogénesis del cáncer renal, generando interés en la repercusión de la diabetes tipo 2 en el pronóstico de la neoplasia. Barone y su grupo ${ }^{18}$ publicaron un metanálisis de 48 artículos, en el
Cuadro 3. Índices de supervivencia

\begin{tabular}{|c|c|c|c|}
\hline \multicolumn{2}{|c|}{ Supervivencia } & Global & LR \\
\hline \multicolumn{2}{|c|}{ General } & 43.5 IC $36.8-50.2$ & 45.3 IC $39.3-51.3$ \\
\hline \multirow{3}{*}{ DM2 } & Con & 35.5 IC 20.9-50.0 & 25.5 IC 11.3-39.6 \\
\hline & Sin & 44.2 IC $37.2-51.2$ & 28.6 IC 22.6-34.6 \\
\hline & $p$ & 0.76 & 0.78 \\
\hline \multirow{3}{*}{ HAS } & Con & 28.5 IC 23.6-33.3 & 22.0 IC 15.5-28.4 \\
\hline & Sin & 43.8 IC 36.5-51.1 & 29.6 IC 23.1-36.2 \\
\hline & $p$ & 0.91 & 0.15 \\
\hline
\end{tabular}

IC: intervalo de confianza; DM2. Diabetes tipo 2; HAS: hipertensión arterial sistémica; LR: supervivencia libre de recurrencia.

que demostraron que $41 \%$ de los pacientes con diabetes tipo 2 preexistente manifiestan tumores malignos, lo que incrementa el riesgo de todas las causas de mortalidad, comparada con los pacientes sin diabetes (RM: 1.41 e IC95\%: 1.241.55). En nuestra serie, la prevalencia de diabetes tipo 2 fue de $18.6 \%$, que coincide con reportes previos que señalan $10-22 . \%$ en pacientes con carcinoma de células renales. ${ }^{19-21}$ Los pacientes con diabetes tipo 2 padecen sobrepeso u obesidad en fases iniciales, además de resistencia a la insulina, integrada por hiperglucemia e hiperinsulinemia, que provoca el aumento de la concentración sérica de IGF-1 y propicia un ambiente favorable para padecer tumores. ${ }^{22,23} \mathrm{Se}$ ha reportado que las células de la carcinoma de

Cuadro 2. Correlación entre diabetes mellitus tipo 2 y la clasificación TNM de cáncer renal

\begin{tabular}{|c|c|c|c|c|c|c|}
\hline \multirow{3}{*}{ Estadio } & \multicolumn{6}{|c|}{ Diabetes mellitus tipo 2} \\
\hline & \multicolumn{2}{|c|}{$\mathbf{T}$} & \multicolumn{2}{|c|}{$\mathbf{N}$} & \multicolumn{2}{|c|}{$\mathbf{M}$} \\
\hline & Con & Sin & Con & Sin & Con & Sin \\
\hline T1a & $17 \%(1)$ & $83 \%(5)$ & - & - & - & - \\
\hline $\mathrm{Tb}$ & $21 \%(3)$ & $79 \%(11)$ & - & - & - & - \\
\hline T2a & $25 \%(3)$ & $75 \%(9)$ & - & - & - & - \\
\hline $\mathrm{T} 2 \mathrm{~b}$ & - & $100 \%(7)$ & $0 \%$ & $100 \%(2)$ & - & - \\
\hline Т3a & - & $100 \%(2)$ & - & - & - & - \\
\hline $\mathrm{T} 4$ & $50 \%(1)$ & $50 \%(1)$ & $0 \%$ & $50 \%(1)$ & $50 \%(1)$ & $50 \%(1)$ \\
\hline $\mathrm{p}$ & \multicolumn{2}{|c|}{0.5} & \multicolumn{2}{|c|}{0.7} & \multicolumn{2}{|c|}{0.5} \\
\hline
\end{tabular}

Clasificación TNM (2009) propuesta por la AJCC (American Joint Committee on Cancer) 
células renales expresan receptores de proliferación celular in vitro para el IGF-1. ${ }^{24}$ En modelos celulares de carcinoma de células renales in vivo, se ha observado que la aplicación del IGF1 estimula el crecimiento tumoral. ${ }^{25}$ Estudios recientes sugieren que el daño acumulado por estrés oxidativo del $A D N$, que incrementa la hiperactivación de Akt/tuberina/mTOR, una de las vías de desarrollo celular del cáncer renal en pacientes con diabetes tipo 2 , tiene función importante en la génesis tumoral. ${ }^{26}$

Larsson y sus colaboradore ${ }^{27}$ demostraron que los pacientes con diabetes tipo 2 tienen mayor riesgo de padecer carcinoma de células renales versus sujetos sanos. Psutka y su grupo, ${ }^{14}$ en su estudio con 1,964 pacientes con carcinoma de células renales localizado, intervenidos quirúrgicamente, reportaron un incremento del riesgo de mortalidad cáncer-específico en sujetos con diabetes tipo 2. La posible repercusión de la diabetes tipo 2 en la mortalidad de pacientes con cáncer renal aún se discute. Algunos estudios de cohorte muestran resultados poco claros, incluso ciertas series han demostrado un efecto negativo de la diabetes tipo 2 en el pronóstico de pacientes con carcinoma de células renales. ${ }^{7,9,14}$ Ha y sus coautores ${ }^{20}$ reportaron, en su serie con 2257 pacientes, menor índice de supervivencia global, supervivencia libre de recurrencia y supervivencia cáncer-específica en sujetos con diabetes tipo 2 y cáncer renal. Sin embargo, diversos estudios italianos contrastan con estos resultados, pues señalan que la diabetes no influye en el pronóstico de pacientes con carcinoma de células renales. La población de esos estudios fue heterogénea e incluyó todos los estadios y tipos histológicos, lo que dificulta concluir de forma certera la influencia de la diabetes tipo 2 en el pronóstico de pacientes con carcinoma de células renales. ${ }^{21}$

En este estudio no encontramos diferencias significativas en la supervivencia global ni cáncer-específica de pacientes con carcinoma de células renales y diabetes tipo 2 versus el grupo control. Sin embargo, se observó una diferencia numérica, con tendencia hacia la disminución de la supervivencia global y libre de recurrencia en pacientes con diabetes tipo 2. Estos datos coinciden con los resultados de diversos estudios publicados. Algunos estudios demuestran que la obesidad, hipertensión arterial y enfermedad renal crónica aumentan el riesgo de carcinoma de células renales. ${ }^{7,28} \mathrm{La}$ hipertensión arterial es común en pacientes con diabetes tipo 2 , incluso afecta a $60 \%$ de estos casos. En nuestra serie encontramos $32.6 \%$, que demuestra su elevada prevalencia en pacientes con carcinoma de células renales. ${ }^{7,20,29} \mathrm{Höfner}$ y su grupo ${ }^{16}$ no encontraron diferencias en el tamaño tumoral de pacientes con y sin diabetes tipo 2, lo que coincide con nuestros resultados. El número de ganglios y metástasis a distancia son factores pronóstico importante; sin embargo, no encontramos diferencias estadísticamente significativas al comparar los pacientes con y sin diabetes tipo 2, estos mismos resultados han sido reportados en grandes series. ${ }^{14}$

Entre las limitaciones de este estudio se encuentran: la naturaleza de ser retrospectivo, el limitado número de pacientes, seguimiento y heterogeneidad de la muestra; no obstante, representa una interesante investigación para reproducirse y tomarse como referencia en otros centros hospitalarios con mayor número de pacientes, principalmente en México, en donde no existen estudios similares, según nuestro conocimiento.

En México, 9.1\% de la población adulta padece diabetes, que se traduce en 6.4 millones de personas, lo que refleja una alta prevalencia e incidencia de diabetes tipo 2 en población mexicana. ${ }^{30}$ Las distintas características fisiopatológicas, genéticas, raciales y ambientales de los pacientes con diabetes tipo 2 pueden afectar el pronóstico del carcinoma de células renales. 


\section{CONCLUSIÓN}

Este estudio demuestra que la diabetes tipo 2 no repercute de forma significativa en la clasificación TNM de pacientes con carcinoma de células renales, ni muestra diferencias significativas en la supervivencia global y libre de recurrencia de sujetos con y sin diabetes tipo 2; sin embargo, suponemos que por el limitado número de pacientes no se obtuvieron resultados similares a los publicados en otras series.

\section{Agradecimientos}

Al Dr. Alejandro García Rodríguez por su invaluable apoyo.

\section{Financiamientos}

Los autores de este artículo declaran no haber recibido algún tipo de financiamiento.

\section{REFERENCIAS}

1. Scosyrev E, Messing J, Noyes K, Veazie P, Messing, E. Surveillance Epidemiology and End Results (SEER) program and population-based research in urologic oncology: An overview. Urol Oncol Semin Orig Investig 2012;30:126-132.

2. Cancer Facts and Figures 2014. [En línea]. Dirección URL: www.cancer.org/research/cancerfactsstatistics/cancerfactsfigures2014/.

3. SINAIS/SINAVE/DGE/SALUD/Perfil epidemiológico de los tumores malignos en México. [En línea]. Dirección URL: http://www.epidemiologia.salud.gob.mx/doctos/infoepid/ publicaciones/2011/monografias/P_EPI_DE_LOS_TUMORES_MALIGNOS_México.pdf. (2016)

4. Navai N, Wood CG. Environmental and modifiable risk factors in renal cell carcinoma. Urol Oncol Semin Orig Investig 2012;30:220-224.

5. Weikert S, Ljungberg B. Contemporary epidemiology of renal cell carcinoma: Perspectives of primary prevention. World J Urol 2010;28:247-252.

6. Sun LM, et al. Hypertension and subsequent genitourinary and gynecologic cancers risk: a population-based cohort study. Medicine (Baltimore) 2015;94(16):e753.

7. Chow WH, Gridley G, Fraumeni JF, Järvholm B. Obesity, hypertension, and the risk of kidney cancer in men. N Engl J Med 2000;343(18):1305-1311.
8. Zelenko Z, Gallagher EJ. Diabetes and cancer. Endocrinol Metab Clin North Am 2014;43(1):167-185.

9. Chen L, Li H, Gu L, Ma X, et al. The impact of diabetes mellitus on renal cell carcinoma prognosis. Medicine (Baltimore) 2015;94(11):1-8.

10. Choi $Y$, Park B, Seo BC, Jeon SS, et al. Body mass index and survival in patients with renal cell carcinoma: $A$ clinical-based cohort and meta-analysis. Int J Cancer 2012;132(3):625-34.

11. Park YH, Lee JK, Kim KM, Kook HR, et al. Visceral obesity in predicting oncologic outcomes of localized renal cell carcinoma. J Urol 2014;192(4):1043-9.

12. Choi, S, Min GE, Jeon SH, Lee HL, et al. Effects of statins on the prognosis of local and locally advanced renal cell carcinoma following nephrectomy. Mol Clin Oncol 2013;1(2):365-368.

13. Tseng C. Type 2 Diabetes Mellitus and Kidney Cancer Risk : A Retrospective Cohort Analysis of the National Health Insurance. PLoS One 2015;10(11):e0142480.

14. Psutka SP, Stewart SB, Boorjian SA, Lohse CM, et al. Diabetes mellitus is independently associated with an increased risk of mortality in patients with clear cell renal cell carcinoma. J Urol 2014;192(6):1620-1627.

15. Lee S, Hong SK, Kwak C, Kim HH, et al. Prognostic significance of diabetes mellitus in localized renal cell carcinoma. Jpn J Clin Oncol 2012;42(4):318-324.

16. Hofner T, Zeier M, Hatiboglu G, Eisen C, et al. The impact of type 2 diabetes on the outcome of localized renal cell carcinoma. World J Urol 2014;32(6):1537-1542.

17. Levey AS, Coresh J, Greene T, Stevens LA, et al. Using standardized serum creatinine values in the modification of diet in renal disease study equation for estimating glomerular filtration rate. Ann Intern Med 2006;145(4):247-54.

18. Barone BB, Yeh HC, Snyder CF, Peairs KS, et al. Long-term all-cause mortality in cancer patients with preexisting diabetes mellitus. JAMA 2008;300(23):2754-2764.

19. Fukushima $\mathrm{H}$, Masuda $\mathrm{H}$, Yokoyama $\mathrm{M}$, Tatokoro $\mathrm{M}$, et al. Diabetes mellitus with obesity is a predictor of recurrence in patients with non-metastatic renal cell carcinoma. Jpn J Clin Oncol 2013;43(7):740-746.

20. Ha YS, Kim WT, Yun SJ, Lee SC, et al. Multi-institutional analysis of localized renal cell carcinoma that demonstrates the impact of diabetic status on prognosis after nephrectomy. Ann Surg Oncol 2013;20(11):3662-3668.

21. Antonelli A, Arrighi N, Corti S, Zanotelli A, et al. Pre-existing type- 2 diabetes is not an adverse prognostic factor in patients with renal cell carcinoma: A single-center retrospective study. Urol Oncol 2012;31(7):1310-1315.

22. Giovannucci E, Michaud D. The role of obesity and related metabolic disturbances in cancers of the colon, prostate, and pancreas. Gastroenterology 2007;132(6):2208-2225.

23. Renehan AG, Frystyk J, Flyvbjerg A. Obesity and cancer risk: the role of the insulin-IGF axis. Trends Endocrinol Metab 2006;17(8):328-336. 
24. Rosendahl A, Forsberg G. Influence of IGF-IR stimulation or blockade on proliferation of human renal cell carcinoma cell lines. Int J Oncol 2004;25(5):1327-1336.

25. Rosendahl AH, Holly JM, Celander M, Forsberg G. Systemic IGF-I administration stimulates the in vivo growth of early, but not advanced, renal cell carcinoma. Int J Cancer 2008;123(6):1286-91.

26. Habib SL, Liang S. Hyperactivation of Akt/mTOR and deficiency in tuberin increased the oxidative DNA damage in kidney cancer patients with diabetes. Oncotarget 2014;5(9):2542-2550.

27. Larsson SC, Wolk A. Diabetes mellitus and incidence of kidney cancer : a meta-analysis of cohort studies. Diabetologia 2001;54(5):1013-1018.
28. Denton MD, MAgee CC, Ovuworie C, Mauiyyedi S, et al. Prevalence of renal cell carcinoma in patients with ESRD pre-transplantation: a pathologic analysis. Kidney Int 2002;61(6):2201-2209.

29. Seo MH, Lee WJ, Park CY, Kim SR, et al. Management of blood pressure in patients with type 2 diabetes meIlitus: a nationwide survey in Korean. Diabetes Metab J 2011;35(4):348-353.

30. Encuesta Nacional en Salud y Nutrición 2012. Instituto Nacional de Salud Pública en México. [En línea]. Dirección URL: <http://ensanut.insp.mx/informes/ENSANUT2012ResultadosNacionales.pdf $>$.

\section{AVISO IMPORTANTE}

La Revista Mexicana de Urología se convierte en una publicación solo digital (www.revistamexicanadeurologia.org.mx) con todas las ventajas que los medios electrónicos ofrecen.

Para consultar el texto completo de los artículos deberá registrarse por una sola vez con su correo electrónico, crear una contraseña, indicar su nombre, apellidos y especialidad.

Esta información es indispensable para saber qué consulta y cuáles son sus intereses, y poder en el futuro inmediato satisfacer sus necesidades de información. 